Sonja Siljak-Yakovlev

\title{
Karyological study of diploid and tetraploid Crepis aurea (Asteraceae)
}

\begin{abstract}
Siljak-Yakovlev, S. 2020: Karyological study of diploid and tetraploid Crepis aurea (Asteraceae) [In Kamari, G., Blanché, C. \& Siljak-Yakovlev, S. (eds), Mediterranean plant karyological data-30]. - Fl. Medit. 30:456-460. http://dx.doi.org/10.7320/F1Medit30.456

Chromosome numbers and karyotypes were studied in eight populations of Crepis aurea from Alps, Dinaric Alps and Apennines. The populations from Alps and Apennines exhibit diploid chromosome number $(2 n=10)$ while the populations from Central Dinaric Alps and one population from Julian Alps were tetraploid $(2 n=4 \mathrm{x}=20)$. For this reason, a detailed comparative analysis of the two cytotypes was undertaken. The hypothesis of auto-polyploidy seems to be the most plausible one. Additionally, it was interesting to deal with the taxonomic status of Crepis aurea var. bosniaca growing in Central Dinaric Alps and whose populations are tetraploid.
\end{abstract}

Keywords: Chromosome number, Crepis aurea subsp. aurea, C. aurea subsp. glabrescens, C. aurea var. bosniaca, karyomorphology, polyploidy.

\section{Introduction}

Crepis aurea (L.) Cass. belongs to the Asteraceae family (sub-family Cichorioideae Chevall., 1828; tribe Cichoriae Lam. \& DC., 1806; sub-tribe Crepidinae Cass., 1827). This is a perennial hemicryptophyte growing in grass and on rocky slopes of the subalpine and alpine regions of South European mountains (from 1000 to $2900 \mathrm{~m}$ ). This species has often changed its taxonomic status, which explains the existence of many synonyms: Leontodon aureus L., 1759; Hieracium aureum (L.) Scop., 1772; Apargia aurea (L.) F.W.Schmidt, 1795; Calliopea aurea (L.) D.Don, 1829; Geracium aureum (L.) Rchb., 1831; Crepis kitaibelii Froel., 1838; Aracium aureum (L.) D.Dietr., 1847; Brachyderea aurea (L.) Sch.Bip., 1866; Hieracioides aureum (L.) Kuntze, 1891. Currently this species comprises three accepted subspecies: Crepis aurea (L.) Cass. subsp. aurea, mainly located in the Alps, Crepis aurea subsp. glabrescens (Caruel) Arcang. located in Apennines, Apuan Alps and mountains of Southwest Balkans, and Crepis aurea subsp. olympica (K. Koch) Lamond which is limited to Turkey.

Of the eight populations studied, four had the diploid $(2 n=10)$ and four the tetraploid $(2 n=4 \mathrm{x}=20)$ chromosome numbers. This led us to make a comparative karyological study of two ploidy levels and to try to understand the origin of polyploidy within Crepis aurea species. Two of these seven populations (one diploid - Col de Galibier and one tetraploid - Mt. Jahorina) are used for karyomorphological analyses. In addition, given that Bosnian populations are all tetraploid, the question arises regarding the real taxonomic status of Crepis aurea var. bosniaca described by Maly (1906). 
1994. Crepis aurea (L.) Cass. subsp. aurea $-2 n=10$ (Figs 1a \& 1b).

Fr: French Alps: Aussois, near to the village, alt. 1450 m, Sept 1980, leg. S. SiljakYakovlev, s.n.

- Col de Galibier, alt. 2200 m, Sept 1980, leg. S. Siljak-Yakovlev, s.n.

- Col de Balme, alt. 2190 m, Aug 1981, leg. D. Cartier \& S. Blaise, s.n.

The material from the Col de Galibier population was used for the fine analysis of the diploid karyotype and construction of a karyogram and idiogram which presents the mean value of the five metaphases belonging to the different individuals (Figs 1, 1a \& 1b). Chromosome types were determined according to the usual recommendation and nomenclature of Levan \& al. (1964).

Karyotype was composed of two metacentric pairs (pairs 4 and 5), one submetacentric (pair 2) and one satellite subtelocentric pair (pair 3). The karyotype formula was as follows: $2 n=4 \mathrm{~m}+2 \mathrm{sm}+2 \mathrm{sm}$-st +2 st-SAT $=10$ chromosomes (Table 1$)$.

Several chromosome counts of $2 n=10$ were previously reported for this taxon (Siljak-Yakovlev 1981, 1982; Lippert \& Heubl 1988; Gemeinholzer 2005; Lippert 2006; Enke \& al 2011).

Table 1. Numerical data of chromosome set for diploid Crepis aurea s. s.

\begin{tabular}{|c|c|c|c|c|c|c|c|}
\hline $\begin{array}{l}\text { Chrom. } \\
\text { pair }\end{array}$ & $\begin{array}{c}\mathrm{L} \\
\text { (in } \mu \mathrm{m})\end{array}$ & $\begin{array}{c}\mathrm{S} \\
(\text { in } \mu \mathrm{m})\end{array}$ & $\begin{array}{c}\text { TL } \\
\text { (in } \mu \mathrm{m})\end{array}$ & RL & $\begin{array}{c}\text { Rapport } \\
\mathrm{L} / \mathrm{S}\end{array}$ & $\begin{array}{c}\text { Centromeric } \\
\text { index }\end{array}$ & $\begin{array}{l}\text { Chrom. } \\
\text { type }\end{array}$ \\
\hline 1 & $\begin{array}{c}3.10 \\
(0.10)^{*}\end{array}$ & $\begin{array}{c}1.02 \\
(0.04)\end{array}$ & 4.12 & $\begin{array}{c}242.7 \\
3\end{array}$ & 3.02 & 24.86 & sm-st \\
\hline 2 & $\begin{array}{c}2.60 \\
(0.09) \\
\end{array}$ & $\begin{array}{c}1.23 \\
(0.05) \\
\end{array}$ & 3.83 & $\begin{array}{c}225.3 \\
1 \\
\end{array}$ & 2.11 & 32.11 & $\mathrm{sm}$ \\
\hline 3 & $\begin{array}{c}2.47 \\
(0.12) \\
\end{array}$ & $\begin{array}{c}0.77 \\
(0.07) \\
\end{array}$ & 3.24 & $\begin{array}{c}190.9 \\
7 \\
\end{array}$ & 3.19 & 23.89 & st-SAT \\
\hline 4 & $\begin{array}{c}1.64 \\
(0.08) \\
\end{array}$ & $\begin{array}{c}1.39 \\
(0.09) \\
\end{array}$ & 3.03 & $\begin{array}{c}178.1 \\
2 \\
\end{array}$ & 1.18 & 45.80 & $\mathrm{~m}$ \\
\hline 5 & $\begin{array}{c}1.63 \\
(0.06) \\
\end{array}$ & $\begin{array}{c}1.14 \\
(0.08)\end{array}$ & 2.77 & $\begin{array}{c}162.8 \\
6 \\
\end{array}$ & 1.43 & 41.11 & $\mathrm{~m}$ \\
\hline
\end{tabular}

*Standard deviation; $\mathrm{L}=$ Long arm; $\mathrm{S}=$ Short arm; $\mathrm{TL}=$ Total length; $\mathrm{RL}=$ Relative length

1995. Crepis aurea subsp. glabrescens (Caruel) Arcang. $-2 n=2 \mathrm{x}=10$.

It: Apennins: Peisey Nancroix, alt. 1700 m, 1983, material from Botanical garden, s.n.

The chromosomal number of $2 n=10$ already reported (Chichiricc \& al. 1979, 1981; Siljak-Yakovlev 1985) has been confirmed again in this work for the population from Apennins. 

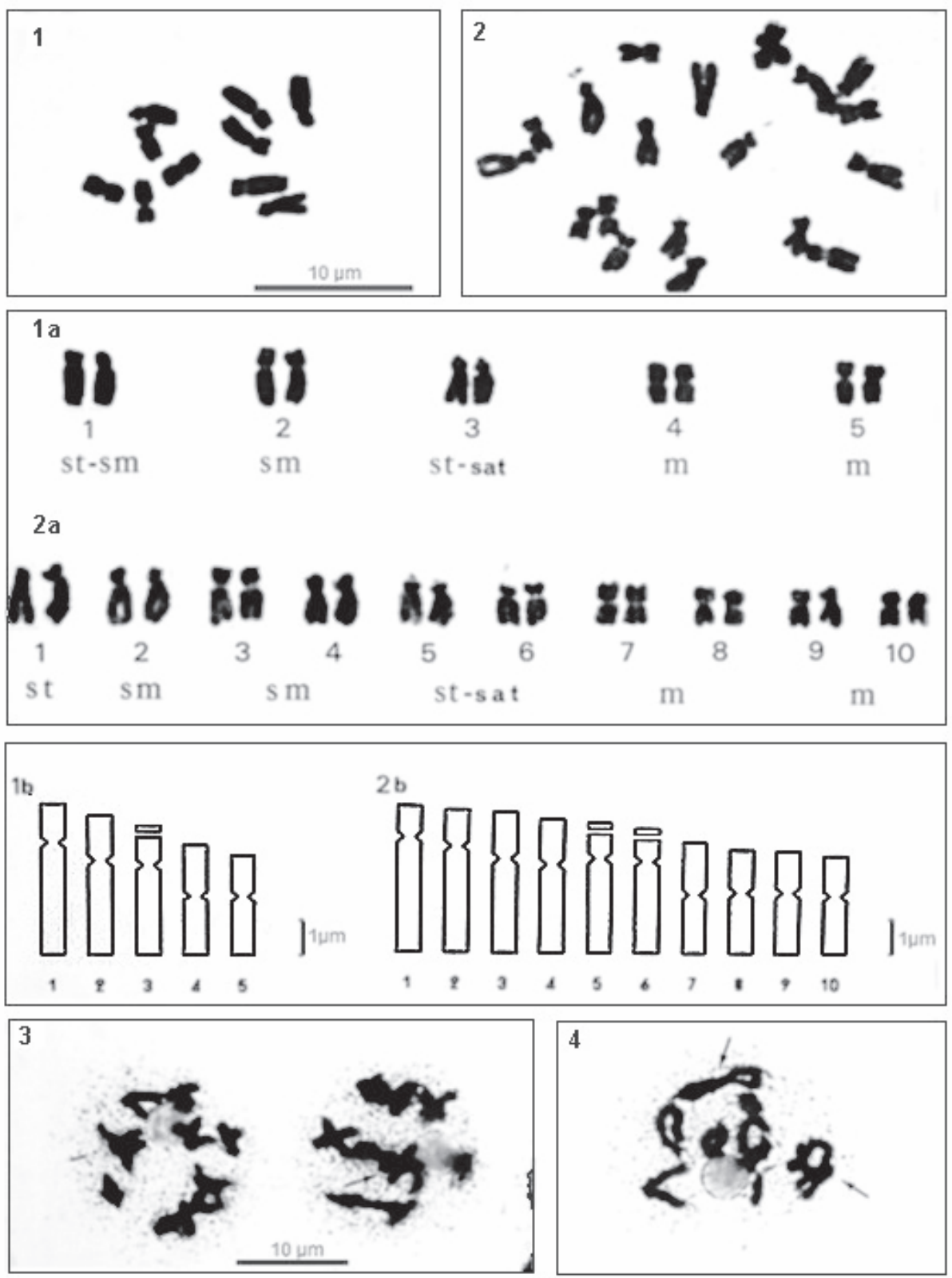

Figs 1-4: 1, Mitotic metaphase chromosome plate of diploid, $2 n=10$, Crepis aurea subsp. aurea from French Alps (Galibier), corresponding karyogram (1a) and ideogram (1b); 2, Metaphase chromosome plate of tetraploid, $2 n=4 \mathrm{x}=20, C$. aurea var. bosniaca from Central Dinaric Alps (Mt. Jahorina), corresponding karyogram (2a) and idiogram (2b); 3 and 4, Meiotic chromosome plates of tetraploid C. aurea var. bosniaca in diakinesis stage. - The rare tetravalents are indicated by arrows. 
1996. Crepis aurea var. bosniaca Maly $-2 n=4 \mathrm{x}=20$ (Figs $2 \mathrm{a}, 2 \mathrm{~b}, 3$ \& 4).

BH: Central Dinaric Alps of Bosnia: Mt. Jahorina, alt. 1600 m, Aug 1979, leg. S. SiljakYakovlev, s.n.

- Central Dinaric Alps of Bosnia: Mt. Treskavica, alt. 1600 m, Aug 1979, leg. S. SiljakYakovlev, s.n.

- Central Dinaric Alps of Bosnia: Mt. Vlasic, alt. 1500 m, Aug 1979, leg. S. SiljakYakovlev, s.n.

Slo: Julian Alps: Mangart, alt. 2100 m, Aug 1981, leg. T. Wraber, s.n.

The tetraploid cytotypes was detected in four populations, three from Bosnia (Central Dinaric Alps) and one from Slovenia (Julian Alps). All these populations grow on limestone while the diploid cytotype was found mainly on granitic substratum. This chromosome number and the new ploidy level were reported for the first time by Siljak-Yakovlev $(1982,1985)$.

For karyological analyses of Crepis aurea var. bosniaca material from population of Mt. Jahorina (Central Dinaric Alps) was used.

Karyotype of this tetraploid cytotypes is composed of four pairs of metacentric chromosomes (pairs 7, 8, 9 and 10), three pairs submetacentrics (pair 2, 3 and 4), and three pairs subtelocentric (pair 1, 5 and 6) from which two bearing the satellites (Figs 2, 2a, 2b; Table 1). The chromosomal formula was as follows: $2 n=4 \mathrm{x}=20=8 \mathrm{~m}+6 \mathrm{sm}+6 \mathrm{st}$ (4 with satellites).

Table 2. Numerical data of chromosome set for tetraploid Crepis aurea var. bosniaca.

\begin{tabular}{|c|c|c|c|c|c|c|c|}
\hline $\begin{array}{c}\text { Chrom. } \\
\text { pair }\end{array}$ & $\begin{array}{c}\mathrm{L} \\
\text { (in } \mu \mathrm{m})\end{array}$ & $\begin{array}{c}\mathrm{S} \\
\text { (in } \mu \mathrm{m})\end{array}$ & $\begin{array}{c}\mathrm{TL} \\
\text { (in } \mu \mathrm{m})\end{array}$ & $\mathrm{RL}$ & $\begin{array}{c}\text { Rapport } \\
\mathrm{L} / \mathrm{S}\end{array}$ & $\begin{array}{c}\text { Centromeric } \\
\text { index }\end{array}$ & $\begin{array}{c}\text { Chrom. } \\
\text { type }\end{array}$ \\
\hline 1 & $\begin{array}{c}3.12 \\
(0.12)^{*}\end{array}$ & $\begin{array}{c}0.85 \\
(0.07)\end{array}$ & 4.05 & 119.77 & 3.79 & 20.86 & $\mathrm{st}$ \\
\hline 2 & $\begin{array}{c}2.96 \\
(0.10)\end{array}$ & $\begin{array}{c}1.02 \\
(0.07)\end{array}$ & 3.98 & 117.55 & 2.90 & 25.65 & $\mathrm{sm}$ \\
\hline 3 & $\begin{array}{c}2.62 \\
(0.13)\end{array}$ & $\begin{array}{c}1.29 \\
(0.04)\end{array}$ & 3.92 & 115.71 & 2.03 & 32.98 & $\mathrm{sm}$ \\
\hline 4 & $\begin{array}{c}1.48 \\
(0.08)\end{array}$ & $\begin{array}{c}1.25 \\
(0.07)\end{array}$ & 3.73 & 110.17 & 1.98 & 33.52 & $\mathrm{sm}$ \\
\hline 5 & $\begin{array}{c}2.67 \\
(0.10)\end{array}$ & $\begin{array}{c}0.67 \\
(0.08)\end{array}$ & 3.33 & 98.47 & 4.00 & 20.00 & st-SAT \\
\hline 6 & $\begin{array}{c}2.58 \\
(0.11)\end{array}$ & $\begin{array}{c}0.60 \\
(0.05)\end{array}$ & 3.19 & 94.17 & 4.28 & 18.95 & $\mathrm{st}-\mathrm{SAT}$ \\
\hline 7 & $\begin{array}{c}1.65 \\
(0.13)\end{array}$ & $\begin{array}{c}1.48 \\
(0.08)\end{array}$ & 3.12 & 92.32 & 1.11 & 47.33 & $\mathrm{~m}$ \\
\hline 8 & $\begin{array}{c}1.71 \\
(0.04)\end{array}$ & $\begin{array}{c}1.19 \\
(0.10)\end{array}$ & 2.90 & 85.55 & 1.44 & 41.01 & $\mathrm{~m}$ \\
\hline 9 & $\begin{array}{c}1.72 \\
(0.13)\end{array}$ & $\begin{array}{c}1.16 \\
(0.05)\end{array}$ & 2.88 & 85.06 & 1.49 & 40.23 & $\mathrm{~m}$ \\
\hline 10 & $\begin{array}{c}1.62 \\
(0.14)\end{array}$ & $\begin{array}{c}1.12 \\
(0.05)\end{array}$ & 2.75 & 81.24 & 1.44 & 40.91 & $\mathrm{~m}$ \\
\hline
\end{tabular}

*Standard deviation; $\mathrm{L}=$ Long arm; $\mathrm{S}=$ Short arm; $\mathrm{TL}=$ Total length; $\mathrm{RL}=$ Relative length 
Comparative analysis of diploid $C$. aurea subsp aurea and tetraploid $C$. aurea var. bosniaca suggests an autopolyploid origin of this last taxon. In fact, var. bosniaca shows a marked clustering of chromosomes by four. Only the first two pairs are of different types which can be the consequence of chromosomal restructuring during diploidization of tetraploid cytotype. Evidence of strong diploidization in var. bosniaca is also expressed by the formation, in the great majority, of the bivalents during the meiosis. Only a few rare tetravalents can be observed in diakinesis (Figs 3 and 4, arrows).

According to all these results it would be legitimate to consider that var. bosniaca merits the rank of a new subspecies within Crepis aurea and that it occupies a part of the area of distribution (mountains of Southwest Balkans) that was previously assigned to $C$. aurea subsp glabrescens.

\section{References}

Chichiricc, G., Frizzi, G. \& Tammaro, F. 1979: Numeri cromosomici per la flora italiana: 652-661. - Inform. Bot. Ital. 11: 3-35.

—, — \& - 1981: Numeri cromosomici per la flora italiana: 652-661. - Inform. Bot. Ital. 11(3): 307314.

Enke, N., Fuchs, J. \& Gemeinholzer, B. 2011: Shrinking genomes? Evidence from genome size variation in Crepis (Compositae). - Pl. Biol. 13: 185-193. https://doi.org/10.1111/j.14388677.2010.00341.x

Gemeinholzer, B. 2005: New chromosome counts for some Lactuceae (Compositae). - Compositae Newslett. 42: 43-46.

Levan, A, Fredga, K, \& Sandberg, A. 1965: Nomenclature for centromeric position on chromosomes. - Hereditas 52(2): 201-220. https://doi.org/10.1111/j.1601-5223.1964.tb01953.x

Lippert, W. 2006: Chromosomenzahlen von Pflanzen aus Bayern und anderen Gebieten. -Ber. Bayer. Bot. Ges. 76: 85-110.

— \& Heubl, G. R. 1988: Chromosomenzahlen von Pflanzen aus Bayern und angrenzenden Gebieten. - Ber. Bayer. Bot. Ges. 59: 13-22.

Maly, K. 1906: Nove biljke iz Bosne I Hercegovine. - Glas. Zem. Muzeja BiH 18: 19-25.

Siljak-Yakovlev, S. 1981: reports [In Love, A. (ed.), IOPB chromosome number reports LXXIII]. Taxon 30: 843-844.

— 1982: reports [In Love, A. (ed.), IOPB chromosome number reports LXXVII]. - Taxon 31: 768.

— 1985: reports [In Love, A. (ed.), IOPB chromosome number reports LXXXVII]. - Taxon 34: 548.

Address of the author:

Sonja Siljak-Yakovlev,

Université Paris-Saclay, CNRS, AgroParisTech, Ecologie Systématique Evolution,

91405 Orsay, France. E-mail: sonia.yakovlev@universite-paris-saclay.fr 